\title{
Independent-of-Delay Stability of Nonlinear Networked Control Systems by Scattering Transformation
}

\author{
Tilemachos Matiakis, Sandra Hirche, and Martin Buss
}

\begin{abstract}
In this paper a novel stability condition is derived for nonlinear networked control systems (NCS) with arbitrarily large constant time delay. The proposed approach is based on input-output models of the subsystems and the scattering transformation, well-known from teleoperation systems analysis with time delay. Passivity of the subsystems has been a prerequisite for guaranteed stability in systems using the scattering transformation. In this paper, the approach is extended to non-passive, non-linear subsystems. The main idea is the compensation of the non-passivity of the plant by excess of passivity of the controller and vice-versa. A numerical example demonstrates that even unstable plants can independently of the constant delay be stabilized over the network.
\end{abstract}

\section{INTRODUCTION}

Due to the increasing complexity of control systems and novel requirements, such as decentralized control, the traditional control architecture with point to point interconnection between the plant and the controller is more and more replaced by Networked Control Systems (NCS). In NCS the plant and the controller are spatially separated and the control loop is physically closed through the communication network as shown in Fig. 1. Compared to traditional control architecture the use of NCS results, among others, in decreased complexity and cost, easier maintenance and system diagnosis, and higher flexibility. Until now specific industrial networks, such as CAN and PROFIBUS, have been mainly used in NCS. Recently, common purpose networks, e.g. Ethernet-like, attract more attention mainly due to their higher flexibility and significantly reduced cost.

However, the transmission of the controller and plant output over a communication network introduces a time delay into the closed loop system, which can be constant or varying. In Ethernet-like networks the time delay depends highly on the concurrent amount of traffic and there are no guarantees for a maximum time delay. Furthermore, in each case, the behavior of the time delay may change dynamically as it depends on the network configuration, e.g. number of nodes. It is well-known that time delay in a closed control loop degrades the performance and can lead to instability. The dynamic behavior of the time delay requires insensitive methodologies with respect to time delay uncertainties in order to guarantee good performance.

T. Matiakis and M. Buss are with the Institute of Automatic Control Engineering, Technische Universität München, D-80290 München, Germany; http://www.lsr.ei.tum.de, T.Matiakis@tum.de M.Buss@ieee.org

$\mathrm{S}$. Hirche is visiting researcher at the Fujita Lab., Dept. of Mechanical and Control Engineering, Tokyo Institute of Technology, 152-8552 Tokyo, Japan,s.hirche@ieee.org

This work was in part supported by the Japanese Society for the Promotion of Science (JSPS) in terms of a Postdoctoral Fellowship for Foreign Researchers granted to S. Hirche.

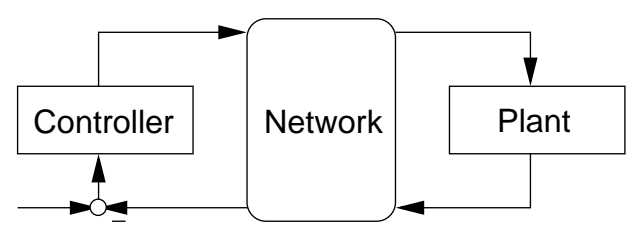

Fig. 1. Networked control system architecture.

Various methodologies have been presented in order to deal with the problem of the time delay in NCS. In [1] the time delay is assumed to be bounded by the sampling period and the stability is analyzed using hybrid system techniques. Random time delays, also bounded by the sampling period, are considered in [2], [3], a stochastic stability analysis is performed and stochastic optimal control applied. A delaydependent approach based on Lyapunov stability with LMI conditions is proposed in [4]. In the seminal work [5], [6] an augmentation technique is applied to transform the time delay system to an augmented discrete system without time delay and a stability condition is presented for periodic time delays. In [7] bounded random time delays are considered and predictive control is applied.

A delay-independent analysis for NCS has been presented in [8] using the notion of scattering transformation. The scattering transformation is applied for the first time in a control context to force feedback telepresence systems in [9], [10] in order to face the problem of stability in the presence of time delay. Using the concepts of passivity and network theory, the derived control law is based upon the fact that a system of interconnected subsystems is passive if each of them is passive, and consequently stable. The scattering transformation passivates the communication subsystem with arbitrarily large constant time delay. However, in it's original version [9], [10] both, the plant and the controller, are required to be passive.

In [8] the scattering transformation is applied for the first time to NCS. A new delay independent stability condition is presented extending the notion of scattering transformation to non-passive systems. Contrary to other methodologies for NCS [1]-[7], the design goals not only for stability and performance, but also for insensitivity of the system with respect to time delay uncertainties are set. In simulation and experiment the proposed approach is insensitive to delay uncertainties and outperforms typical approaches for time delay systems like the Smith predictor and a PI controller.

However, like in most of the NCS literature a linear time-invariant (LTI) system is considered. In this paper a new delay independent stability condition is derived for the 
general nonlinear case, extending scattering transformation to non-passive, non-linear systems. Unlike common approaches of non-linear systems with time delay which make use or Lyapunov-Krasovskii of Razumikhin Theorem thereby requiring a state-space representation of the system, in this approach an input-output stability analysis is performed based on the concepts of passivity and $L_{2}$ gain. The proposed approach can deal even with unstable plants as demonstrated in a numerical example.

This paper is organized as follows: Section II introduces the notions of $L_{2}$ stability, passivity and scattering transformation; a novel stability condition is given in Section III together with the system description. Some observations concerning passivity, $L_{2}$ stability and the scattering transformation are presented in Section IV. A numerical example is presented in Section $\mathrm{V}$, followed by conclusions in Section VI.

\section{THEORETICAL BACKGROUND}

\section{A. $L_{2}$ Stability}

Let $\|u\|_{L_{2}}$ denote the $L_{2}$ norm of a piecewise squareintegrable function $u(\cdot): \mathbb{R}_{+} \rightarrow \mathbb{R}^{m}$ with $\mathbb{R}_{+}$is the set of non-negative real numbers and $\mathbb{R}^{m}$ the Euclidean space of dimension $m$. The truncation of $u(\cdot)$ up to the time $t$ is denoted by $u_{t}(\cdot)$, and the extended space of Lebesgue integrable functions by $L_{2 e}$.

A system is said to be finite gain $L_{2}$ stable if there are some constants $\gamma, \beta \geq 0$ such that between the input $u(\cdot) \in L_{2 e}$ the output $y(\cdot) \in L_{2 e}$ of the system the following inequality holds [11]

$$
\left\|y_{t}\right\|_{L_{2}} \leq \gamma\left\|u_{t}\right\|_{L_{2}}+\beta \quad \forall u, \quad t \in[0, \infty) .
$$

The smallest possible value $\gamma$, for which a $\beta$ exists, such that (1) is satisfied, is the $L_{2}$ gain of the system. The $L_{2}$ stability condition states, that each bounded $L_{2}$ norm input signal is mapped to a bounded $L_{2}$ norm output signal.

\section{B. Input-Feedforward-Output-Feedback-Passivity}

A system is said to be Input-Feedforward-OutputFeedback-Passive (IF-OFP) if

$$
\int_{0}^{t} u(\tau) y(\tau) \mathrm{d} \tau \geq \delta\left\|u_{t}\right\|_{L_{2}}^{2}+\varepsilon\left\|y_{t}\right\|_{L_{2}}^{2}-E_{0}, \quad \forall t>0, u \in L_{2 e}
$$

holds, where $u(\tau) y(\tau)$ represents the instantaneous power input to the system, $E_{0}$ is the initially stored energy in the system, and $\delta, \varepsilon \in \mathbb{R}$ are constants. The above inputoutput description can be seen as a generalization of the passivity concept, i.e. if $\delta=\varepsilon=0$ then the system is passive, meaning that it cannot generate energy. If $\delta=0$ and $\varepsilon>0$ the system is called output feedback strictly passive and if $\delta>0$ and $\varepsilon=0$ input feedforward strictly passive. In both these cases the system dissipates energy. If one or both of the values $\delta, \varepsilon$ are negative then there is a shortage of passivity in the system. In other words, the system can generate energy, but this energy is bounded by the squared $L_{2}$ norm of the input and/or the output signal.

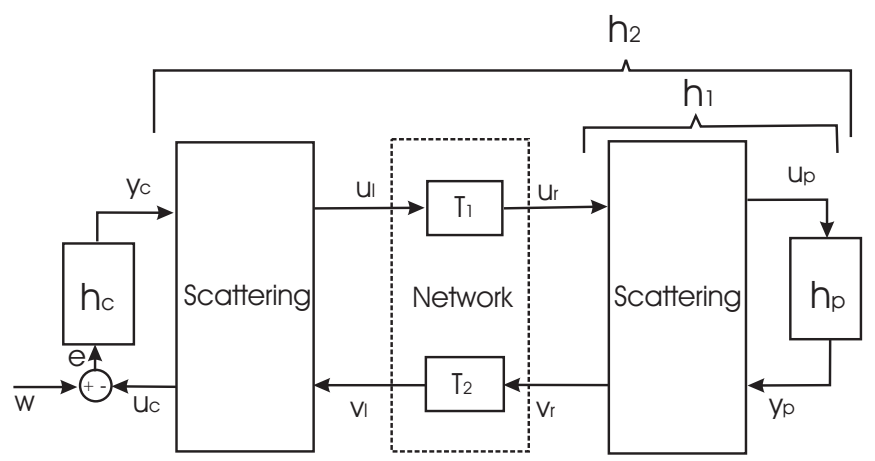

Fig. 2. NCS with scattering transformation.

One important feature of IF-OFP is that under certain conditions it can guarantee stability of systems combined in feedback interconnection, i.e. the next proposition holds

Proposition 1 [11]: A system comprising two subsystems which satisfy (2) for some $\delta_{i}, \varepsilon_{i}, i \in\{c, p\}$, combined in feedback interconnection, is finite gain $L_{2}$ stable if

$$
\varepsilon_{c}+\delta_{p}>0 \quad \text { and } \quad \varepsilon_{p}+\delta_{c}>0
$$

What is interesting in the above case is that some of the $\delta_{i}, \varepsilon_{i}$ can be also negative, as long as the above conditions are satisfied. This is the case, where shortage of passivity of the plant is compensated by the excess passivity of the controller, or vice-versa.

\section{Scattering Transformation}

The scattering transformation is used in order to passivate the communication network with arbitrary large constant time delay. In case of time delay, the communication network can be modelled as a time delaying two-port with time delays $T_{1}, T_{2}$ in the forward and the backward path respectively, as shown in Fig. 2.

The time-delaying two-port generates energy as shown in [9], i.e. the passivity condition is violated. The scattering transformation, placed in front and behind of the network, see Fig. 2, is used in order to passivate the communication network with the transformation equations given by

$$
\begin{array}{ll}
u_{l}=\frac{1}{\sqrt{2 b}}\left(u_{c}+b y_{c}\right) ; & u_{r}=\frac{1}{\sqrt{2 b}}\left(y_{p}+b u_{p}\right) ; \\
v_{l}=\frac{1}{\sqrt{2 b}}\left(u_{c}-b y_{c}\right) ; & v_{r}=\frac{1}{\sqrt{2 b}}\left(y_{p}-b u_{p}\right)
\end{array}
$$

where $b>0, u_{p}, y_{p}$ the plant input and output, $y_{c}, u_{c}$ the left side communication subsystem input and output and $u_{r}, v_{r}, u_{l}, v_{l}$ the values transmitted over the communication channel. The indices $(\cdot)_{l},(\cdot)_{r}$ denote the lefthand and righthand side of the communication channel. The value $u_{l}$ is transmitted over the forward channel and arrives delayed by the time delay $T_{1}$ at the plant, now denoted by $u_{r}$. Analogously, the value $v_{r}$ is transmitted over the backward channel with the time delay $T_{2}$, hence

$$
u_{r}(t)=u_{l}\left(t-T_{1}\right) \quad \text { and } \quad v_{l}(t)=v_{r}\left(t-T_{2}\right) .
$$


The time delays are constant but arbitrarily large. The energy balance for the two-port is now computed with the input and output vectors respectively

$$
u^{T}=\left[\begin{array}{ll}
y_{c} & -y_{p}
\end{array}\right], \quad y^{T}=\left[\begin{array}{ll}
u_{c} & u_{p}
\end{array}\right]
$$

and the reformulated transformation equations (3)

$$
\begin{aligned}
\int_{0}^{t} u^{T} y \mathrm{~d} \tau & =\int_{0}^{t}\left(u_{c} y_{c}-u_{p} y_{p}\right) \mathrm{d} \tau \\
& =\frac{1}{2} \int_{0}^{t}\left(u_{l}^{2}-u_{r}^{2}+v_{r}^{2}-v_{l}^{2}\right) \mathrm{d} \tau \\
& =\frac{1}{2} \int_{t-T_{1}}^{t} u_{l}^{2} \mathrm{~d} \tau+\frac{1}{2} \int_{t-T_{2}}^{t} v_{r}^{2} \mathrm{~d} \tau \geq 0 \quad \forall t .
\end{aligned}
$$

The passivity condition, (2) for $\delta, \varepsilon=0$, is satisfied, hence the communication two-port with constant time delay is passive.

\section{Stability AnAlysis}

\section{A. System Description}

We consider a system consisting of a SISO plant and a SISO controller, see Fig. 2. The plant is described by a mapping $h_{p}$ from the plant input $u_{p}$ to the plant output $y_{p}$

$$
h_{p}: L_{2 e} \rightarrow L_{2 e},
$$

with the energetic input-output behavior described by (2) with $\delta_{p}, \varepsilon_{p}$, and $E_{p, 0}$, i.e.

$$
\int_{0}^{t} u_{p}(\tau) y_{p}(\tau) \mathrm{d} \tau \geq \delta_{p}\left\|u_{p, t}\right\|_{L_{2}}^{2}+\varepsilon_{p}\left\|y_{p, t}\right\|_{L_{2}}^{2}-E_{p, 0} \quad \forall t>0 .
$$

Analogously, the controller input-output behavior is described by a mapping $h_{c}$ from the control error $e=w-u_{c}, w$ being the desired value and $u_{c}$ the lefthand side output of the communication channel, to the output $y_{c}$

$$
h_{c}: L_{2 e} \rightarrow L_{2 e},
$$

with the energetic input-output behavior described by (2) with $\delta_{c}, \varepsilon_{c}$, and $E_{c, 0}$.

The plant is connected to the controller by the communication subsystem including the communication network described by (4), with constant but arbitrary large time delays $T_{1}, T_{2}$ and the scattering transformation given by (3). Without loss of generality we assume that there is no energy initially stored in the communication, hence

$$
u_{r}(t)=0 \quad \forall t \in\left[0, T_{1}\right) ; \quad v_{l}(t)=0 \quad \forall t \in\left[0, T_{2}\right) .
$$

The subsystem $h_{1}$, see Fig. 2, is defined to be the mapping from the input $u_{r}$ to the output $v_{r}$ of the right part scattering transformation, i.e. $h_{1}$ contains the plant and right hand scattering transformation. The subsystem $h_{2}$ is defined to be the mapping from the input $y_{c}$ to the output $u_{c}$ of the left side of the communication subsystem, i.e. $h_{2}$ contains the plant and the whole communication subsystem.

\section{B. Preliminaries}

Before presenting the stability results we will give a corollary and introduce a notion in accordance to finite gain $L_{2}$ stability (1), both necessary for the ongoing analysis.

Corollary 1: In IF-OFP systems (2) without loss of generality we can consider

$$
\varepsilon \delta \leq 1 / 4
$$

Proof: If $\delta \varepsilon>1 / 4$ degenerate cases occur. There are two cases to consider : (a) $\delta, \varepsilon>0$, and (b) $\delta, \varepsilon<0$. In the first case, using the square complement, (2) can be reformulated to

$$
\lambda(t)=\int_{0}^{t}\left(\varepsilon y-\frac{u}{2}\right)^{2}+u^{2}\left(\delta \varepsilon-\frac{1}{4}\right) \mathrm{d} \tau \leq \varepsilon E_{0}
$$

which cannot be satisfied for any system and each $u$, since $\lambda(t)$ can grow indefinitely depending only on the input signal $u$ if $(\delta \varepsilon-1 / 4)>0$. Analogously, in the second case (b) the reformulation of (2) leads to $\lambda(t) \geq \varepsilon E_{0}$. This is true for any pair $u(\tau), y(\tau)$ since $\varepsilon E_{0} \leq 0$ and $\lambda(t) \geq 0$, thus does not impose any restriction to the system input-output behavior.

In accordance to finite gain $L_{2}$ stability (1) we will denote as finite gain $L_{2}$ square stable, a system satisfying

$$
\left\|y_{t}\right\|_{L_{2}}^{2} \leq \gamma^{2}\left\|u_{t}\right\|_{L_{2}}^{2}+\beta^{2} \quad \forall u, \quad t \in[0, \infty) .
$$

for some $\beta, \gamma>0$. Condition (8) is necessary and sufficient for finite gain $L_{2}$ stability. Sufficiency can be seen by taking the square root of (8) and substituting inequality $\sqrt{a^{2}+b^{2}} \leq a+b, a, b>0$ in the right part of (8). Necessity can be seen by taking the square of (1) and substituting inequality $(a+b)^{2} \leq 2 a^{2}+2 b^{2}$. However the gains in (1), (8) are not the same.

\section{Stability with Passive Plant and Controller}

If the plant is passive, using the sign notation of the input and output vectors given in (5), $h_{2}$ can be seen as feedback interconnection of passive subsystems (plant and passivated communication subsystem) and is thus passive. Further, the controller $h_{c}$ is assumed to be passive. The two passive subsystems $h_{2}$ and $h_{c}$ are again in feedback interconnection, thus the closed loop is passive and consequently, under observability assumptions, stable for arbitrary large constant time delay. In fact, all the passivity theorems [11] and the corresponding stability results can be applied. The controller and the plant can be either linear or nonlinear, continuous or discrete time, time-invariant or time-varying subsystems. The plant parameters can be even uncertain as long as the plant remains passive. However, the passivity requirements might be too restrictive in real applications.

\section{Stability with Non-passive Plant or Controller}

In [8] it is shown that the scattering transformation can guarantee stability even if the plant or the controller are non-passive LTI systems. Here, the more general, nonlinear case is considered and it is shown how shortage of passivity 
of the plant can be compensated by excess of passivity of the controller and vice-versa, so that stability can be guaranteed independently of delay even for non-passive, nonlinear plants. More specifically the following theorem holds:

\section{Theorem 2: If}

$\delta_{c}+\varepsilon_{p}>0, \quad \varepsilon_{c}+\varepsilon_{p} b^{2}>0, \quad$ in case $\quad \alpha=\varepsilon_{p} b-\frac{\delta_{p}}{b}<0$ and

$\delta_{c}+\frac{\delta_{p}}{b^{2}}>0, \quad \varepsilon_{c}+\delta_{p}>0, \quad$ in case $\quad \alpha \geq 0$

where $b>0$ the scattering transformation parameter, the closed loop system with the scattering transformation the plant and the controller, is finite gain $L_{2}$ stable.

Proof: For the proof Proposition 1, the scattering transformation equations (3) and some mathematical manipulation are applied. Substituting the right part of the reformulated equations (3) in (6), for the $h_{1}$ subsystem seen in Fig. 2 holds

$$
\begin{aligned}
& \left(1-\frac{\delta_{p}}{b}-\varepsilon_{p} b\right)\left\|u_{r, t}\right\|_{L_{2}}^{2}-\left(1+\frac{\delta_{p}}{b}+\varepsilon_{p} b\right)\left\|v_{r, t}\right\|_{L_{2}}^{2} \geq \\
& \alpha \int_{0}^{t} 2 u_{r} v_{r} \mathrm{~d} \tau-2 E_{p, 0} \quad \forall t>0,
\end{aligned}
$$

Two cases are to consider depending on the sign of $\alpha$. In case $\alpha<0$ inequality $u_{r}^{2}+v_{r}^{2} \geq 2 u_{r} v_{r}$ by multiplication gives $2 u_{r} v_{r} \alpha \geq\left(u_{r}^{2}+v_{r}^{2}\right) \alpha$ and by substituting in the right part of (9) we get

$$
\left(1-2 \varepsilon_{p} b\right)\left\|u_{r, t}\right\|_{L_{2}}^{2}-\left(1+2 \varepsilon_{p} b\right)\left\|v_{r, t}\right\|_{L_{2}}^{2} \geq-2 E_{p, 0}, \quad \forall t>0 .
$$

If $\quad \alpha \geq 0, \quad$ inequality $\quad 2 u_{r} v_{r} \geq-u_{r}^{2}-v_{r}^{2}$ gives $2 u_{r} v_{r} \alpha \geq-\left(u_{r}^{2}+v_{r}^{2}\right) \alpha$ and like before by substituting in the right part of (9) we get

$$
\left(1-\frac{2 \delta_{p}}{b}\right)\left\|u_{r, t}\right\|_{L_{2}}^{2}-\left(1+\frac{2 \delta_{p}}{b}\right)\left\|v_{r, t}\right\|_{L_{2}}^{2} \geq-2 E_{p, 0}, \quad \forall t>0 .
$$

In the next, first the case where $\alpha<0$ is considered. From $\alpha<0$ and Corollary 1 it is easy to see that $\left|\varepsilon_{p} b\right|<1 / 2$ so $1-2 \varepsilon_{p} b>0$ and $1+2 \varepsilon_{p} b>0$. By taking further into account that the constant time delay operator satisfies (8) for gain $\gamma=1$, i.e. accordingly with assumption (7)

$$
\begin{aligned}
& \left\|u_{r, t}\right\|_{L_{2}}^{2} \leq\left\|u_{l, t}\right\|_{L_{2}}^{2}, \\
& \left\|v_{l, t}\right\|_{L_{2}}^{2} \leq\left\|v_{r, t}\right\|_{L_{2}}^{2}, \quad \forall t>0
\end{aligned}
$$

holds, it follows that

$$
\left(1-2 \varepsilon_{p} b\right)\left\|u_{l, t}\right\|_{L_{2}}^{2}-\left(1+2 \varepsilon_{p} b\right)\left\|v_{l, t}\right\|_{L_{2}}^{2} \geq-2 E_{p, 0} .
$$

Equation (11), by substituting the left part of the scattering equations gives for the $h_{2}$ subsystem seen in Fig. 2

$$
\int_{0}^{t} y_{c} u_{c} \mathrm{~d} \tau \geq \varepsilon_{p} b^{2}\left\|y_{c, t}\right\|_{L_{2}}^{2}+\varepsilon_{p}\left\|u_{c, t}\right\|_{L_{2}}^{2}-E_{p, 0}
$$

Thus, for the subsystem $h_{2}$, with input $y_{c}$ and the output $u_{c}$, (2) holds with $\delta=\varepsilon_{p} b^{2}, \varepsilon=\varepsilon_{p}$. In case $\alpha \geq 0$, following a similar procedure, it is proved that for $h_{2}$, (2) holds for $\delta=\delta_{p}$ and $\varepsilon=\delta_{p} / b^{2}$. The proof is completed by applying directly to the above Proposition 1 .

Concluding the above, it is seen that if (2) holds for the plant for some $\delta_{p}, \varepsilon_{p}$, then for the subsystem $h_{2}$, (2) also holds, with $\delta=\varepsilon_{p} b^{2}$ and $\varepsilon=\varepsilon_{p}$, or $\delta=\delta_{p}$ and $\varepsilon=\delta_{p} / b^{2}$ depending on the sign of $\alpha$.

Remark 1: The effect of the scattering transformation is to preserve the input output behavior of the plant as described by (2) to the subsystem $h_{2}$, independently of the constant time delay, but for more conservative values than the initial $\delta_{p}, \varepsilon_{p}$. This result comes from the fact that the scattering transformation transforms an IF-OFP system to a system described by (8). The arbitrary large time delay does not change this gain in (8) since the constant time delay operator satisfies also (8) with $\gamma=1$. Then, applying the left part of the scattering transformation the subsystem $h_{2}$ is described again by (2) but for more conservative values than the initial $\delta_{p}, \varepsilon_{p}$. The time delay operator could be replaced by any other with gain $\gamma=1$ in (8), and the above analysis should be exactly the same. The exact relation between the $\delta, \varepsilon$ and $\gamma$ will be given in the next section.

Remark 2: Although in the case of a passive system where $\delta=\varepsilon=0$ the $b$ parameter does not affect the energetic properties of $h_{2}$ and can be chosen freely, if $\delta, \varepsilon \neq 0, b$ should be tuned appropriately in order to guarantee stability.

Remark 3: With Theorem 2 not only non-passive plants can be stabilized with the scattering transformation, but also in case of a strictly passive plant, a non-passive controller would be allowed, as it can be seen from Theorem 2 and positive $\delta_{p}, \varepsilon_{p}$. A non-passive controller should possibly lead to less conservative design.

\section{IF-OFP, SQUARE $L_{2}$ GAIN AND THE SCATTERING TRANSFORMATION}

The scattering transformation can be seen as a transformation between an IF-OFP system and a system described by (8). As shown in Section III, assuming a system satisfying (2) for $\varepsilon_{p} \delta_{p} \leq 1 / 4$ and $\alpha<0$, after substituting the right part of the reformulated scattering equations (3), (10) is reached which can be rewritten as

$$
\left\|v_{r, t}\right\|_{L_{2}}^{2} \leq \frac{1-2 \varepsilon_{p} b}{1+2 \varepsilon_{p} b}\left\|u_{r, t}\right\|_{L_{2}}^{2}+\frac{2 E_{p, 0}}{\left(1+2 \varepsilon_{p} b\right)}, \quad \forall t \geq 0,
$$

where $u_{r}, v_{r}$ is the input and output of the subsystem $h_{1}$. If $\alpha \geq 0$, by the same procedure we reach

$$
\left\|v_{r, t}\right\|_{L_{2}}^{2} \leq \frac{1-2 \delta_{p} / b}{1+2 \delta_{p} / b}\left\|u_{r, t}\right\|_{L_{2}}^{2}+\frac{2 E_{p, 0}}{\left(1+2 \delta_{p} / b\right)}, \quad \forall t \geq 0
$$

Thus subsystem $h_{1}$ satisfies (8) for $\gamma^{2}=\frac{1-2 \varepsilon_{p} b}{1+2 \varepsilon_{p} b}$ or $\gamma^{2}=\frac{1-2 \delta_{p} / b}{1+2 \delta_{p} / b}$ depending on the sign of $\alpha$. In the case of a passive system, i.e. $\delta_{p}=\varepsilon_{p}=0, \gamma=1$, if $\delta_{p}<0$ 
or $\varepsilon_{p}<0, \gamma>1$ and in case the system is strictly passive, i.e. $\delta_{p}, \varepsilon_{p}>0, \gamma<1$. Thus, the scattering transformation transforms an IF-OFP system to a system described by (8), and as the passivity becomes more or less strict, the gain $\gamma$ becomes smaller or larger respectively.

Going the other way around, the subsystem with input $u_{l}$ and output $v_{l}$, see Fig. 2, is considered which is assumed to satisfy (8) for some $\gamma, \beta \geq 0$. By substituting the left part of the scattering transformation equations in (8) and after some mathematical manipulation we get

$$
\int_{0}^{t} y_{c} u_{c} \mathrm{~d} \tau \geq \frac{b}{2} \frac{1-\gamma^{2}}{1+\gamma^{2}}\left\|y_{c, t}\right\|_{L_{2}}^{2}+\frac{1}{2 b} \frac{1-\gamma^{2}}{1+\gamma^{2}}\left\|u_{c, t}\right\|_{L_{2}}^{2}-\frac{\beta^{2}}{1+\gamma^{2}}
$$

where $y_{c}, u_{c}$ is the input and the output respectively, of the subsystem $h_{2}$. Thus, $h_{2}$ satisfies (2) with $\delta=\frac{b}{2} \frac{1-\gamma^{2}}{1+\gamma^{2}}$ and $\varepsilon=\frac{1}{2 b} \frac{1-\gamma^{2}}{1+\gamma^{2}}$. So a system described by (8) is transformed to an IF-OFP system and the smaller the gain is the more strictly passive the system becomes. In case $\gamma=1$ a passive system is recovered which is the original case of the scattering transformation with passive subsystems.

Concluding the above, the scattering transformation transforms a IF-OFP system to a systems described by (8) and vice versa, and the smaller the gain is in (8) the more strictly passive the system becomes.

\section{Numerical ExAmple}

In the next we proceed with some simulations in order to test the efficacy of the above analysis. A spring-massdamper system is considered, with nonlinear spring coefficient $K\left(x_{1}\right)=2 x_{1}^{2} \mathrm{kgm} / \mathrm{s}^{2}$ where $x_{1}$ is the displacement, mass $M=1 \mathrm{~kg}$ and negative damping $D=-4 \mathrm{kgm}$ seen in Fig. 3.

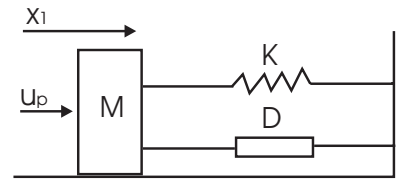

Fig. 3. Mass spring damper system with nonlinear spring coefficient and negative damping.

The input $u_{p}$ to the system is the applied force to the mass and the output is the velocity of the mass. If $x_{2}=\frac{\mathrm{d} x_{1}}{\mathrm{~d} t}$ is the velocity, the state space equations become

$$
\dot{x}_{1}=x_{2}, \quad \dot{x}_{2}=-2 x_{1}^{3}+4 x_{2}+u_{p}, \quad y_{p}=x_{2}
$$

The above system is initially unstable because of the negative damper coefficient. The goal is to stabilize it through a network with constant time delay. A zero input $w=0$ is considered in the controller and the initial conditions of the plant are $x_{1}(0)=0, x_{2}(0)=1$.

Taking as a storage function $V(x)=2 x_{1}^{4}+(1 / 2) x_{2}^{2}$ we have $\dot{V}=-4 x_{2}+u_{p} y_{p} \quad$ and by integrating from 0 to $t, \int_{0}^{t} u_{p} y_{p} \mathrm{~d} \tau \geq-4 y_{p}^{2}-V(x(0)), \quad$ so is satisfied for $\delta=0, \varepsilon=-4$. A proportional
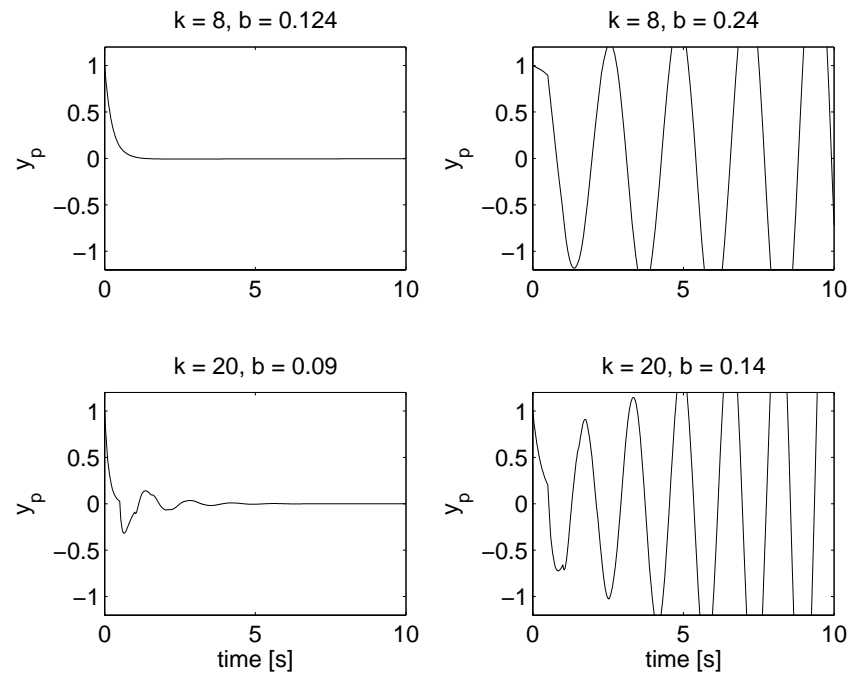

Fig. 4. Response of the nonlinear plant for different values of the controller $k$ and the parameter $b$.

controller is also used, $y_{c}=k u_{c}$. For the proportional controller $\quad u_{c} y_{c}=k u_{c}^{2}=\gamma k u_{c}^{2}+\frac{1-\gamma}{k} y_{c}^{2} \quad$ for $\quad 0<\gamma<1$, thus (2) is satisfied for $\delta_{c}=\gamma k, \varepsilon_{c}=\frac{1-\gamma}{k}$ and $0<\gamma<1$. Because $\varepsilon_{p} b-\delta_{p} / b=-4 b<0$, from Theorem 2, stability of the closed loop system is guaranteed as long as

$$
\gamma k-4>0
$$

and

$$
\frac{1-\gamma}{k}-4 b^{2}>0 \text {. }
$$

Equation (14) gives $k>4$ and $4 / k<\gamma<1$ and (15) by substituting the less conservative value for $\gamma=4 / k$, gives

$$
b<\frac{\sqrt{k-4}}{2 k} .
$$

The response of the closed loop system with zero input $w=0$ is tested with a forward and backward time delay of $T_{1}=T_{2}=250 \mathrm{~ms}$. Two values for the controller are checked $k=8$ and $k=20$. The results are presented in Fig. 4.

In the first case, where $k=8$, (16) gives $b<0.125$. The response is indeed stable for $b=0.124$ as the velocity settles to 0 and becomes unstable for approximately $b=0.24$. In the second case, (16) gives $b<0.1$. The response becomes unstable for approximately $b=0.14$.

In the next the response of the system with and without the scattering transformation are compared for the above two different values of the controller and different values of the time delay. In the case where $k=8$ and $b=0.124$ four different values of the roundtrip time delay are tested, $T \in[50,100,150,500] \mathrm{ms}$ divided in equal parts in forward and backward time delay. The results are presented in Fig. 5. From Fig. 5 it is seen that the closed loop system without the scattering transformation is sensitive to the time delay $T$. For $T>100 \mathrm{~ms}$ the response is affected significantly and for $T \geq 150 \mathrm{~ms}$ it is unstable. On the other hand the system with the scattering transformation, not 

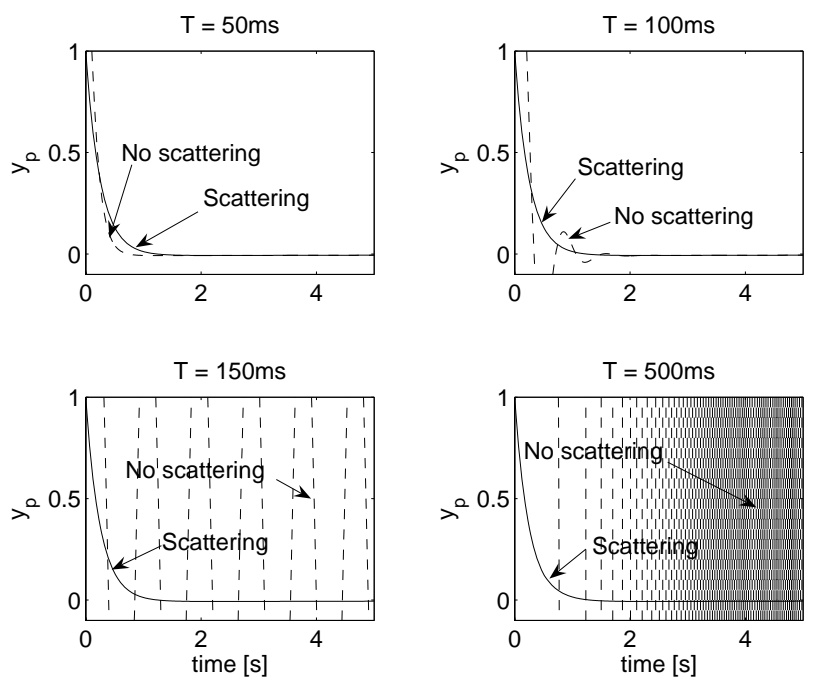

Fig. 5. Response of the system with and without the scattering transformation for $k=8, b=0.124$ and different values of the time delay.
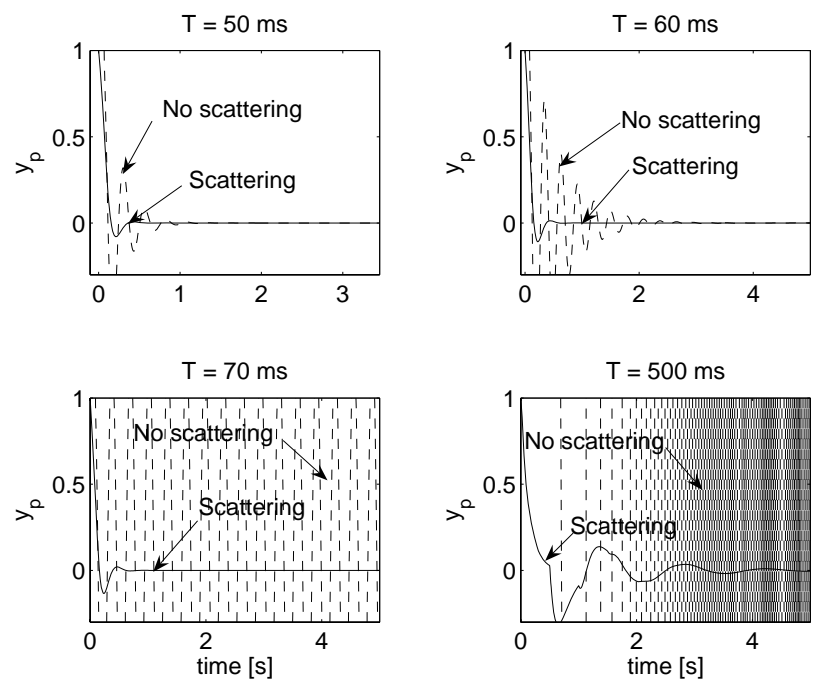

Fig. 6. Response of the system with and without the scattering transformation for $k=20, b=0.09$ and different values of the time delay.

only remains stable independently of the time delay, but it's response is not much affected with the increase of the time delay, even for large values, as seen in the last figure for $T=500 \mathrm{~ms}$.

In Fig. 6 the results for the second controller, $k=20$ are presented. Again four values of the roundtrip time delay are tested $T \in[50,60,70,500] \mathrm{ms}$ divided in equal parts in the forward and backward time delay. With greater controller gain the closed loop system without the scattering transformation is even more sensitive to time delay, as it becomes unstable for approximately $T=70 \mathrm{~ms}$. The system with the scattering transformation however, like before, gives a steady response independently of the time delay. For large values some oscillations appear, as seen for the case of $T=500 \mathrm{~ms}$. In both cases, $k=8$ and $k=20, x_{1}$ settles also to zero but it is not shown here due to space limitations.

From the above it is concluded that the scattering transformation can be used to control even unstable, non-passive and nonlinear plants through a network, while stability is guaranteed independently of the time delay. Furthermore, in this case the response of the system with the scattering transformation is not only stable, but it is not affected much from variations of the time delay, thus the closed loop system is insensitive to time delay uncertainties.

\section{CONCLUSIONS}

In this paper a novel delay-independent approach for nonlinear NCS is proposed using the scattering transformation. Recently, it has been shown that the scattering transformation can be used in networked control systems in order to guarantee independent-of-delay stability in the case of non-passive, LTI plants. In this paper the more general nonlinear case is considered and a novel independent of delay stability condition is established. The underlying principle is the compensation of the shortage of passivity of the plant by excess passivity of the controller, and vice versa. The connection between the IF-OFP, square $L_{2}$ gain and the scattering transformation is also given. In an example with a unstable nonlinear mass-spring-damper system the proposed approach not only guarantees stability independent of time delay, but makes also the system insensitive to time delay uncertainties.

\section{ACKNOWLEDGMENTS}

Detailed comments by the anonymous reviewers are highly appreciated.

\section{REFERENCES}

[1] W. Zhang, M. S. Branicky, and S. M. Philips, "Stability of network control systems," IEEE Control Systems Magazine, vol. 21, no. 1, pp. 84-99, February 2001.

[2] J. Nilsson, "Real-time control systems with delays," Ph.D. dissertation, Department of Automatic Control, Lund Institute of Technology, 1998.

[3] M. Mukai and M. Fujita, "On Networked LQG Control with Varying Delay using the Delta Operator," in Proceedings of the 44th SICE Annual Conference (SICE2005), Okayama, Japan, 2005, pp. 34433448.

[4] M. Yu, L. Wang, and T. Chu, "An LMI approach to robust stabilization of networked control systems," in Proceedings of the 16th IFAC World Congress, July 2005.

[5] Y. Halevi and A. Ray, "Integrated communication and control systems: Part I analysis," Journal of Dynamic Systems, Measurement and Control, vol. 110, pp. 367-373, 1988.

[6] _ - "Integrated communication and control systems: Part II design considerations," Journal of Dynamic Systems, Measurement and Control, vol. 110, pp. 374-381, 1988.

[7] G. P. Liu, J. X. Mu, and D. Rees, "Networked predictive control of systems with random network transmission delay - a polynomial approach," in Proceedings of the 16th IFAC World Congress, July 2005.

[8] T. Matiakis, S. Hirche, and M. Buss, "The scattering transformation for networked control systems," in Proceedings of 2005 IEEE Conference on Control Applications, August 2005, pp. 705-710.

[9] R. J. Anderson and M. W. Spong, "Bilateral control of teleoperators with time delay," IEEE Transactions on Automatic Control, vol. 34, no. 5, pp. 494-501, May 1989.

[10] G. Niemeyer and J. Slotine, "Stable adaptive teleoperation," International Journal of Oceanic Engineering, vol. 16, no. 1, pp. 152-162, 1991.

[11] H. K. Khalil, Nonlinear Systems, P. Hall, Ed., 1996. 